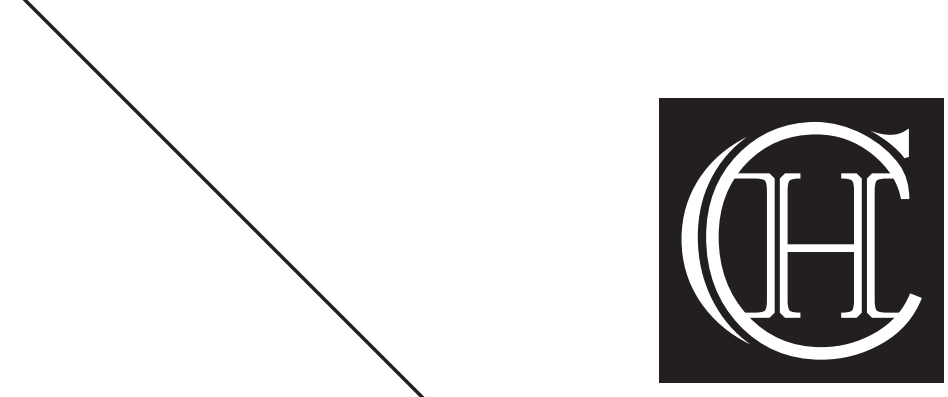

COMUNICACIÓNyHOMBRE

\title{
ESTUDIOS
}

STUDIES 

Dra. Lucía Caro-Castaño

Universidad de Cádiz, Cádiz, España

@ lucia.caro@uca.es

(iD 0000-0003-2720-1534

Dr. David Selva-Ruiz

Universidad de Cádiz, Cádiz, España
- Recibido / Received 7 de octubre de 2019

- Aceptado / Acepted 20 de octubre de 2019

- Páginas / Pages De la 35 a la 57

n ISSN: 1885-365X

\section{Data storytelling: el empleo de datos en la construcción de relatos publicitarios de marca}

Data storytelling: the use of data in the construction of brand advertising stories

\section{Resumen}

Se aborda la contribución de los datos a la construcción de relatos de marca. Se analiza, pues, cómo el storytelling, potenciado por el uso de datos, puede ayudar a la generación de engagement en el consu-midor y a la construcción de mensajes publicitarios creativos, recurriendo a la categoría "Creative Data Lions" del festival publicitario Cannes Lions, y, en particular, a todas las campañas seleccionadas en la sección "Data storytelling". Los resultados permiten observar que se trata de una tendencia enormemen-te diversa, cumpliendo los datos roles muy diversos en su relación con el storytelling.

PALABRAS CLAVE: data storytelling, creative data, datos, storytelling, engagement, creatividad, publicidad.

\section{Abstract}

This paper addresses the contribution of data to the construction of brand stories. It is analyzed, thus, how storytelling, enhanced by the use of data, can help generating consumer engagement and constructing creative advertising messages. To that end, "Creative Data Lions" category - and, in particular, all the campaigns selected in "Data storytelling" section - of Cannes Lions advertising festival is used. The results allow us to observe that this is an enormously diverse trend, with data fulfilling very diverse roles in relation to storytelling.

KEY WORDS: music emotion recognition, emotions, music moods, affective computing. 


\section{Introducción}

«Dear Cannes, it's time to expect more from technology». Este era el texto que rezaba en una valla de IBM en las inmediaciones del Palais des Festivals en mayo de 2019 (Cannes Lions, 2019b). La tecnológica insistía con esta pieza -integrada en una iniciativa below the line basada en inteligencia artificial ${ }^{1}$ - en uno de sus valores centrales de marca, al tiempo que enviaba un mensaje muy claro a toda la profesión en su festival de referencia acerca la estrecha comunión del binomio publicidad y tecnología. En realidad, este binomio siempre ha mantenido una estrecha relación, dado el creciente valor que lo tecnológico tiene en las sociedades modernas en términos de estatus (Marwick, 2013) y a la fascinación que despierta en el ser humano toda nueva tecnología que medie de modo significativo su experiencia de la realidad (Miller y Sinanan, 2014; McLuhan, 1980).

Esta capacidad de fascinación ha sido siempre muy apreciada por la publicidad: ya que se trata de un mensaje (potencialmente) intrusivo, lo mínimo es intentar que al receptor le resulte divertido o interesante (McKee y Gerace, 2018; Wagler, 2013; Roberts, 2005). Y si el mensaje no es nuevo o atractivo per se, que el modo de comunicarlo sí lo sea, funcionando así la tecnología como gancho para atraer la atención y generar engagement. En este sentido, la profesión ha asistido durante los últimos 25 años a todo un proceso de redefinición de su estructura, buscando cómo integrar la tecnología y a sus expertos en sus procesos de creación de piezas (Stuhlfaut y Windels, 2019).

La redefinición de la estructura de la agencia corre pareja $-y$ en buena medida responde a- cambios de gran calado producidos por el proceso de dataficación propio del ecosistema de plataformas web y dispositivos digitales (Van Dijck y Poell, 2016; 2013; Van Dijck, 2014; Mayer-Schönberger y Cukier, 2013; Boyd y Crawford, 2012). Dicho proceso ha permitido sustanciales avances en la construcción de perfiles de usuario cada vez más sofisticados para la personalización de los servicios digitales y la navegación en la web (Cufoglu, 2014), así como un uso más adecuado de las posibilidades expresivas de los medios interactivos (Hook, 2018; Solana, 2012; Truong, McColl y Kitchen, 2010) para el desarrollo de campañas y acciones de marca. Así, el uso de datos estaría permitiendo que la profesión avance en su relación con la tecnología en un sentido que supera la fascinación artificiosa por lo nuevo para conectar de un modo más significativo y relevante con sus públicos de interés e incluso crear nuevos servicios y productos que aportan un valor que va más allá de la comunicación. Como se verá en el análisis de los trabajos seleccionados en la sección "Data storytelling" que se realiza en este trabajo, la incorporación tanto de macrodatos como de microdatos a los mensajes de marca está permitiendo desarrollar campañas donde los datos son empleados como recurso creativo para enriquecer el storytelling de la marca, pero también en campañas donde el storytelling es utilizado como recurso creativo capaz de humanizar y hacer relevantes estos datos.

\footnotetext{
1/ Se trata de una colaboración con Cannes Lions denominada "Talks @ Cannes" y es una plataforma que permite la búsqueda entre los vídeos de las charlas de las ediciones 2018 y 2019 mediante lenguaje natural. Las recomendaciones del sistema llevan al usuario a las escenas más útiles de los vídeos, ofreciéndole una experiencia personalizada. La acción puede consultarse en: https://www.ibm.com/ watson-advertising/news/cannes-2019
} 


\section{Objetivo y metodología}

Este trabajo tiene como objetivo examinar la contribución de los datos a la construcción de relatos publicitarios de marca mediante el análisis de campañas de éxito. Se evalúa, pues, la interrelación entre el storytelling y los datos, y cómo esta puede ayudar a la generación de engagement en el consumidor y a la construcción de mensajes publicitarios creativos.

Para alcanzar este objetivo, se lleva a cabo, por una parte, una revisión de la relación existente entre datos y storytelling a la hora de desarrollar mensajes de marca significativos, prestando atención a aspectos como la personalización, el uso de la dataficación, o las estrategias narrativas posibles; y por otra, un análisis cualitativo de campañas de éxito seleccionadas por el festival publicitario Cannes Lions en su categoría "Creative Data Lions" y, en particular, en su sección "Data Storytelling".

La muestra está compuesta por las 44 campañas que han sido incluidas en la short list - es decir, seleccionadas como candidatas a obtener un premio - de la sección "Data Storytelling" integrada en la categoría "Creative Data Lions". Esta categoría nace en el año 2015 como consecuencia del auge de los datos en la creatividad publicitaria. De este modo, se han analizado 44 campañas correspondientes a 5 años -10 en 2015, 8 en 2016, 10 en 2017, 9 en 2018, y 7 en 2019-. No se ha tenido en cuenta, pues, si finalmente obtuvieron premio o no, puesto que la propia inclusión en la short list se considera ya una señal de éxito.

Estas 44 campañas no pueden ser consideradas como representativas del discurso publicitario, fundamentalmente por dos motivos. En primer lugar, los festivales no son un reflejo neutro de la realidad publicitaria, sino una muestra de la mejor y más avanzada publicidad. En segundo, el uso de datos en publicidad es ya una tendencia suficientemente relevante como para tener una categoría propia -y con tanta importancia como podrían tener las más tradicionales de "Film Lions", "Print \& Publishing Lions", etc. -, pero no podemos considerarla generalizada en el ámbito publicitario, sino algo que aún muestra ciertos tintes experimentales. Sin embargo, el objetivo de este trabajo no es crear una radiografía del statu quo del discurso publicitario, sino analizar las posibilidades de un nuevo fenómeno. Y estas campañas analizadas no hacen sino dibujarnos estos primeros caminos posibles que hasta la fecha no han dejado de crecer.

\section{Datos, storytelling y la creación de mensajes de marca significativos}

La posibilidad de disponer de los datos de millones de individuos de modo individualizado, en los que puede combinarse información sociodemográfica, actitudinal y comportamental 
(Cufoglu, 2014; Turow, 2011), además de plantear un gigantesco reto social y político², ofrece un nuevo mundo de posibilidades comunicativas y creativas para la interlocución de la marca con sus públicos. Si, como plantean Heding, Knudtzen y Bjerre, la marca es un narrador (storyteller) del que se espera que juegue un papel como artefacto cultural dentro de la sociedad de consumo (Heding, Knudtzen y Bjerre en Fernández Gómez, Rubio-Hernández y Pineda, 2019: 26), el uso creativo de los datos del consumidor permite ahora la creación de historias de marca a partir de las experiencias, intereses y características reales de ese consumidor: significados de marca colectivos en los que el sujeto no solo puede proyectarse, sino de los que puede formar parte - mediante la incorporación de sus datos-.

\subsection{Personalización para conectar con el consumidor}

La extensión de la lógica de la personalización en el ecosistema de medios digital (Couldry y Turow, 2014), así como la normalización del consumo audiovisual individual a demanda (Álvarez Monzonzillo, 2013), han favorecido que el consumidor muestre una actitud cada vez más negativa ante los mensajes que siguen una dinámica (McKee y Gerace, 2018; Turow, 2011; Truong, McColl y Kitchen, 2010), la propia de los formatos publicitarios tradicionales como la cuña de radio o el spot televisivo: contenidos que interrumpen al espectador. En este sentido, se calcula que un $45 \%$ de la población mundial de usuarios de la web estaría utilizando programas de bloqueo en sus equipos para evitar los formatos de publicidad intrusiva (Gómez Nieto, 2018).

Ante este rechazo creciente a los formatos push (Wagler, 2013), los profesionales de la publicidad han venido desarrollando durante las dos últimas décadas nuevas formas de interpelar a sus públicos adoptando estrategias con un enfoque de atracción (pull). Así, puede observarse un progresivo crecimiento en la aparición de formatos centrados en construir mensajes de marca que buscan ser percibidos como contenido, sobre todo de entretenimiento (branded content), ofrecer información útil (publicidad nativa), o formatos capaces de incorporar la información de marca en un contexto en el que pueda prestar un servicio al consumidor (búsquedas pagadas, apps de marca, etc.). En definitiva, ante la fragmentación de las audiencias y su progresiva capacidad para controlar qué contenido consumen y cuándo, las marcas vienen trabajando en diferentes fórmulas para transformar sus mensajes en contenidos y/o servicios útiles para el consumidor (Feng y Ots, 2018; Dahlen y Rosengren, 2016), siendo la personalización del mensaje de marca la estrategia central (Selva-Ruiz y Caro-Castaño, 2016; Couldry y Turrow, 2014).

\footnotetext{
2/ Queda fuera del horizonte de este trabajo el análisis crítico de las cuestiones éticas y legales que abre este proceso de dataficación en términos de privacidad y vigilancia. A este respecto pueden revisarse los trabajos de McStay (2016), Van Dijck (2014) y Boyd y Crawford (2012).
} 


\subsection{Posibilidades de la dataficación para el trabajo creativo PUBLICITARIO}

De acuerdo con Mayer-Schönberger y Cukier, "dataficar un fenómeno es ponerlo en un formato que lo cuantifica de modo que pueda ser tabulado y analizado» (2013: 78). En definitiva, dataficar es transformar los datos en un recurso que luego podrá ser utilizado para obtener valor económico de estos datos (Van Dijck y Poell, 2013, 2016). El actual diseño del ecosistema digital ha sido desarrollado para transformar en dataficable cualquier dimensión de la existencia: opiniones políticas, preferencias musicales, rutinas cotidianas geolocalizadas gracias a los metadatos del teléfono móvil, red de contactos, etc. De este modo, y por primera vez en la historia, las relaciones, experiencias y emociones de los individuos pueden ser almacenadas, cuantificadas y analizadas para la elaboración de perfiles de usuario (microtargeting), pudiendo ser empleados estos perfiles para la analítica predictiva y en tiempo real (Van Dijck y Poell, 2013). Esto es algo que habría sido utilizado, por ejemplo, por Cambridge Analytica en su trabajo como consultora en el referéndum sobre el Brexit o en la campaña de Donald Trump a las presidenciales norteamericanas de 2016, gracias a los datos obtenidos a través de Facebook (González, 2017; Grassegger y Krogerus, 2017; Mavriki y Karida, 2017).

La evolución y perfeccionamiento de la compra programática (Carrillo-Durán, RodríguezSilgado, 2018) unida a la disponibilidad de estos perfiles multidimensionales permite a anunciantes y agencias construir mensajes personalizados (ad targeting), prediseñando diferentes versiones de las piezas de acuerdo con la variedad de perfiles a los que se enviará el mensaje: cambios en la paleta de colores, elección de la celebridad más adecuada de acuerdo con sus preferencias en las redes sociales, adaptación de los aspectos textuales, etc. Esta capacidad de adaptación a las características psicográficas y de contexto del usuario provoca incluso preocupación entre los consumidores, que pueden sentirse vigilados y controlados, especialmente en aquellos casos en los que el anuncio incorpora información relativa a su historial de navegación (Jung, 2017; Zhu y Chang, 2016), si bien, cuando el usuario percibe el mensaje como relevante, el rechazo al anuncio tiende a pasar a un segundo plano (Jung, 2017).

Además del microtargeting multidimensional y la personalización del mensaje, el tercer uso que la publicidad puede hacer de los datos consiste en su utilización como recurso narrativo dentro de la historia que se quiere contar. Como se verá en algunas de las campañas seleccionadas en la sección "Data Storytelling" de Cannes Lions, la pieza publicitaria puede en la actualidad ser concebida para interpelar a un sujeto concreto, construyendo un relato que incorpora al individuo a través de los datos automáticamente generados por utilización de los medios y dispositivos digitales (plataformas de medios sociales, smartphones, pulseras de control biométrico, etc.). Así, aunque el formato de difusión del contenido publicitario final pueda seguir una dinámica push (mailing digital, mensajería móvil, etc.), el grado de personalización del mensaje y una correcta utilización de los recursos narrativos pueden lograr una alta aceptación e involucración del consumidor con la marca. A continuación se revisa la noción de storytelling y sus posibles usos en relación con los datos. 


\subsection{Aproximación teórica al Data storytelling}

\subsubsection{El giro narrativo}

Christian Salmon explica el storytelling como una forma de discurso basada en el arte de contar historias que atraviesa todos los ámbitos de la sociedad actual estableciendo «engranajes narrativos según los cuales los individuos son conducidos a identificarse con unos modelos y conformarse con unos protocolos» (Salmon, 2008: 38). Siguiendo con el enfoque del autor: «la realidad está ahora envuelta por una red narrativa que filtra las percepciones y estimula las emociones útiles» (2008: 38). En la misma línea, aunque desde un enfoque funcionalista y celebratorio de esta capacidad para moldear percepciones y emociones, McKee y Gerace (2018) plantean la necesidad de que las marcas transformen los datos en historias a través de cualquiera de sus comunicaciones de marketing, relaciones públicas y publicidad, dado el poder que las técnicas del storytelling aportarían al mundo empresarial en términos de creación de sentido; es lo que denominan como storynomics:

La diferencia entre los datos y la historia es esta: los datos enumeran lo que ha pasado; la historia expresa cómo y por qué ha sucedido. Los datos compilan hechos por cantidad y frecuencia; la historia revela las causas que hay detrás y debajo de estos hechos. La historia elimina lo irrelevante, se concentra en la dinámica del cambio para volver luego a darle forma a la cuestión dentro de una estructura que enlaza los eventos encadenando causa y efecto en el tiempo (McKee y Gerace, 2018).

Abundando en esta idea, y centrándola en el empleo del storytelling en publicidad, cabe destacar la apreciación que realizaba Roig en el prólogo al libro de Salmon (2008) al indicar que la estrategia esencial sobre la que se apoya esta técnica es el deseo de todo sujeto de protagonizar una gran historia, de sentirse protagonista de una biografía intensa: «sobre esta vulnerabilidad se apoya la estrategia del storytelling» (2008: 17). Esto es algo que la publicidad ha venido explotando desde el desarrollo de la investigación motivacional y su énfasis en el self-concept, hasta sus actuales reformulaciones dentro de una perspectiva más relacional como la que propone el branding cultural (Fernández Gómez, Rubio-Hernández \& Pineda, 2019). Este último enfoque de brand management entiende que las marcas más poderosas son concebidas y gestionadas como repositorios globales de la identidad (individual y colectiva):

Las teorías culturales de las marcas parten del «imaginario colectivo» de Jung, de la arquetípica como retrato universal y sintético, de los relatos y narraciones que contienen de forma atractiva y renovada tales arquetipos [...] o ideas colectivas y, por supuesto, de la mitología como fuente de inspiración y madre de todas las historias. El branding cultural entiende que el consumidor demanda historias que le ayudan a ser él mismo y que lo proyectan respecto a los demás (2019: 33).

Desde esta perspectiva, el storytelling es observado como una estrategia de gran utilidad 
para las marcas y sus mensajes, en tanto que, al apostar por un estilo emocional y actualizar mitos culturalmente reconocibles, favorecen la creación de experiencias dentro de una determinada cosmovisión en la que el consumidor puede proyectarse y sentirse partícipe. La incorporación de datos automáticamente generados por el usuario a estos relatos de marca a través de la dataficación del ecosistema digital y los dispositivos de cuantificación del yo (Gilmore, 2016), permitiría llevar estos procesos psicológicos de proyección a un nivel aún más significativo para los consumidores y fans de la marca.

\subsubsection{Storytelling y datos}

Para lograr una integración de los datos y su potencial narrativo en el desarrollo del mensaje publicitario -yendo así más allá del uso meramente adaptativo a las características psicodemográficas, contextuales y comportamentales del ad targeting - ha sido necesaria la incorporación de tecnólogos especializados en los equipos creativos. En este sentido cabe destacar los resultados del estudio de Stuhlfaut y Windels (2019) acerca de la evolución de la estructura de las principales agencias de publicidad norteamericanas. De acuerdo con los investigadores, la tecnología ha pasado de ser observada como parte de la fase de producción a ser sistemáticamente incorporada dentro del proceso creativo, como explicaba uno de los directores creativos entrevistados: «nuestros tecnólogos creativos están ahí para ayudar con las ideas. Ya hemos superado aquello de presentar ideas al cliente que luego no pueden ser desarrolladas» (2019: 12). De este modo, y de acuerdo con sus conclusiones, en la actualidad la dupla creativa estaría siendo ampliada con la incorporación de especialistas en tecnología que intervienen desde la fase inicial de definición del concepto creativo (2019: 17-18).

La innovación narrativa que posibilitan las nuevas fuentes de datos automatizadas y las potencialidades del vídeo interactivo aportarían al menos dos tipos de beneficios de acuerdo con Hook (2018: 43):

- $\quad$ presentar los datos mediante historias interactivas puede «ofrecer medios alternativos que revelen, contextualicen y expliquen los datos»;

- equipar a los cineastas para que puedan utilizar estas nuevas fuentes de datos en la creación de historias audiovisuales puede producir nuevas experiencias mediáticas para el espectador.

En su análisis de una muestra de 43 casos de estudio de vídeos orientados a datos, el investigador encontró diferentes tipos de fuentes de datos y niveles de personalización. En relación con el grado de personalización de la experiencia para el espectador, Hook halló que en la mayoría de los casos $(60,5 \%)$ los datos empleados eran iguales para toda la audiencia, si bien el $34,5 \%$ sí utilizaba datos individuales. En estos casos, las categorías de datos empleados fueron: medios sociales, localización, dispositivo de visualización, datos sobre hábitos de consumo del espectador (emails y consumo de música, por ejemplo) y datos procedentes de dispositivos del internet de las cosas (2018: 49). El 23,3\% restante de la muestra hacía un uso híbrido entre datos individuales y compartidos. En estos casos el 
conjunto de datos empleado era el mismo para todos los espectadores, si bien se ofrecían ejemplos con datos individuales para exponer un enfoque personal de estos datos (2018: 49).

Otro hallazgo interesante del estudio de Hook es que más de la mitad de la muestra $(58,1 \%)$ empleaba datos extraídos de manera dinámica mientras el espectador veía y/o interactuaba con el vídeo gracias al empleo de APIs abiertas o utilizando datos disponibles a través del servidor del propio usuario (2018: 49). Este es un modo de generar experiencias audiovisuales basadas en datos que ha podido constatarse en trabajos anteriores tanto en la creación de experiencias de marca como de videoclips (Selva-Ruiz y Caro-Castaño, 2016; Caro-Castaño y Selva-Ruiz, 2011).

Hook encontró en su análisis diez estrategias en las que se combina el storytelling audiovisual con el uso de datos (Tabla 1), siendo la principal la estrategia de contextualización - aparece en 30 de los 43 casos - , al ser capaz de poner en conexión al usuario con aquellos aspectos de los datos con los que pueden sentirse más directamente interpelados (2018: 51).

Tabla 1. Estrategias narrativas en el uso de datos

\begin{tabular}{|l|l|}
\hline Contextualización & $\begin{array}{l}\text { El vídeo contextualiza la relevancia de la fuente } \\
\text { de datos }\end{array}$ \\
\hline $\begin{array}{l}\text { Datos personales como dispositivo } \\
\text { narrativo }\end{array}$ & $\begin{array}{l}\text { La personalización amplifica la intención del } \\
\text { cineasta }\end{array}$ \\
\hline Vídeo que crea intriga & La pieza crea intriga acerca de los datos \\
\hline Ficción convertida en realidad & $\begin{array}{l}\text { Ilustra inicialmente un escenario de ficción que } \\
\text { luego es demostrado como una propuesta con } \\
\text { visos de realidad empleando diferentes fuentes } \\
\text { de datos }\end{array}$ \\
\hline Datos de los iguales como recurso & $\begin{array}{l}\text { Los datos capturados de otros espectadores apo- } \\
\text { yan la intención y el mensaje del cineasta }\end{array}$ \\
\hline Uso de personajes & $\begin{array}{l}\text { El personaje favorece la involucración del espec- } \\
\text { tador con los datos }\end{array}$ \\
\hline Datos como estructura narrativa & $\begin{array}{l}\text { Los datos ofrecen un camino para navegar a } \\
\text { través del vídeo o la serie de piezas }\end{array}$ \\
\hline Datos para aportar viveza (liveness) & $\begin{array}{l}\text { El empleo de datos dinámicos provee de una } \\
\text { sensación de contemporaneidad al vídeo }\end{array}$ \\
\hline Uno de atmósferas cinematográficas & $\begin{array}{l}\text { Las atmósferas cinematográficas re-enmarcan la } \\
\text { experiencia del usuario respecto a los datos }\end{array}$ \\
\hline & $\begin{array}{l}\text { La interacción con los datos como un recurso } \\
\text { que favorece la intención del emisor }\end{array}$ \\
\hline
\end{tabular}

Fuente: Hook, 2018

Finalmente, y en relación con el uso que las marcas pueden hacer del data storytelling 
más allá del formato vídeo, Holland (2019) propone a las empresas cuatro formas de contar historias con datos:

- Celebrar la relación con el cliente con historias personalizadas. Holland (2019) ejemplifica esta estrategia con la acción de marketing que Spotify lanzó en diciembre de 2017 y actualizó en 2018: Spotify Wrapped ${ }^{3}$. Se trata de una lista de reproducción de las canciones más escuchadas por el usuario durante ese año, incorporando un resumen de los cinco artistas y las cinco canciones que más escuchó, la cantidad de minutos de consumo y el género más frecuente.

- Convertir la experiencia del cliente en una historia de datos. Muchas marcas emplean la visualización de los datos de actividad del cliente como un modo de mostrar su evolución. Esto es algo que el sector bancario viene ofreciendo a sus clientes mediante sus herramientas online, permitiendo al usuario la visualización de la evolución de sus ahorros y gastos, comparativas entre periodos temporales, la posibilidad de fijarse objetivos y observar su grado de cumplimiento, etc.

- Simplificar datos complejos con storytelling visual. Pese a la accesibilidad de muchas de las fuentes de datos autogenerados, es necesario dedicar tiempo y tener ciertos conocimientos para comprender estos datos en bruto. De ahí que exista un amplio campo de investigación e importantes esfuerzos por parte de los gobiernos en torno a la visualización de datos para favorecer acercamientos más atractivos e intuitivos (Eberhardt y Silveira, 2018). Esto es algo en lo que vienen innovando especialmente instituciones y organismos de gobierno, que se apoyan en el storytelling visual para acercar a la ciudadanía a los resultados que arrojan los datos abiertos sobre el funcionamiento de sistemas complejos como la red de transportes de su ciudad o el consumo de energía del país ${ }^{4}$. Como se verá a continuación, varias de las campañas seleccionadas en la sección "Data Storytelling" son fruto de este esfuerzo de instituciones y gobiernos por humanizar los datos.

- Conectar a la marca con los retos de sus clientes. Otro de los usos propuestos por Holland (2019), especialmente indicado para el mercado del B2B, consiste en la creación de acciones por parte de la marca en las que emplee los datos para demostrar a sus clientes que comprende sus necesidades y retos mediante piezas o iniciativas en las que emplean datos reales de sus ámbitos de actividad.

3/ Esta campaña forma parte de la muestra analizada en este trabajo. El usuario puede acceder a esta información personalizada sobre el año 2018 en el siguiente enlace: https://spotifywrapped.com 4/ A este respecto, se recomienda revisar la iniciativa de visualización de datos puesta en marcha por el Gobierno de Canadá: https://open.canada.ca/en/data-visualization 


\section{Data storytelling en campañas publicitarias}

\section{1. “Data Storytelling” en el festival Cannes Lions}

Cannes Lions International Festival of Creativity es considerado como el principal festival publicitario de ámbito global. Esto, que resulta algo consabido en el sector, puede evidenciarse en datos, puesto que es el primer festival en número de premios entregados, número de delegados inscritos, número de entradas venidas, número de países participantes, etc. Ser uno de los primeros festivales publicitarios - nació en 1954- no le ha privado de evolucionar junto a la publicidad misma. Si en su nacimiento era esencialmente un festival de spots, progresivamente fue dando entrada a otras categorías - prensa, exterior, radio, etc. - , y el número de categorías no ha dejado de crecer, siendo 27 en la actualidad.

Una de las últimas categorías en llegar a Cannes Lions es "Creative Data", que ha sido la elegida para este trabajo. Su nacimiento tiene lugar en el reciente año de 2015, de modo que solo ha estado presente en las últimas 5 ediciones del festival. El abstract de esta categoría afirma lo siguiente:

Los "Creative Data Lions" celebran la interrelación entre las ideas y la información. Las entradas en la categoría "Creative Data" deben demostrar claramente cómo la ejecución/campaña fue mejorada o impulsada por el uso, la interpretación, el análisis o la aplicación creativa de datos. El uso creativo de datos debe estar presente en el núcleo de la idea y los resultados o el impacto deben ser claros y robustos (Cannes Lions, 2019a).

Como el resto de categorías del festival, "Creative Data" se subdivide en diversas secciones: "Data-enhanced Creativity", "Data-driven Targeting", "Data-driven Consumer Product", "Data Storytelling", "Data Visualisation", "Data Technology", "Use of Real-time Data", "Social Data \& Insight", "Creative Data Collection \& Research" y "Data Integration". Este trabajo se centra en la sección "Data Storytelling", dado que es la que conecta de un modo más certero con el objetivo marcado. El abstract de esta sección prácticamente define el objeto de estudio de este trabajo:

Campañas en las que los datos han contribuido a exitosas historias de marca/ consumidor. Las entradas deben mostrar cómo la historia fue desarrollada a través de datos de cara a generar un engagement significativo en el consumidor o cómo los mensajes derivados de los datos fueron presentados para proveer una narrativa de marca potente. Se incluye el periodismo de datos (Cannes Lions, 2019).

Aunque se abundará en esta cuestión más adelante, la definición de la categoría, siendo bastante clara, es a la vez amplia y diversa. Así, da cabida tanto a la instrumentalización de los datos para generar historias atractivas para el público como al empleo de la creatividad y el storytelling para comunicar datos de forma atractiva.

Entre las 44 campañas que han sido incluidas en las short lists de los 5 años de existencia 
de esta sección, pueden encontrarse campañas con anunciantes pertenecientes a sectores muy diversos y creadas por agencias norteamericanas, europeas, oceánicas, sudamericanas y asiáticas.

\subsection{Análisis de las campañas}

De acuerdo con el análisis que se ha llevado a cabo respecto a las 44 campañas seleccionadas, pueden observarse los siguientes rasgos en el data storytelling:

- Concepto laxo de narración.

- Relación bidireccional entre datos y storytelling.

- Diversidad en cuanto a tipos de datos.

- La agencia como pivote.

- $\quad$ Automatización vs acción humana.

- Diversidad e interconexión entre diversas tecnologías y herramientas.

- Aportación de valor al usuario.

- Relación con insights y experiencias cotidianas y con la agenda pública.

- Tendencia a la metadiscursividad.

En un análisis de campañas que concurren en una sección de "Data Storytelling", lo primero que llama la atención es el empleo de un concepto tremendamente laxo de narración. Por ejemplo, si se parte de los postulados del Grupo de Entrevernes (1982), una narración implicaría, simplificando, una transición entre estados. Y efectivamente podemos observar este rasgo en algunas de las campañas analizadas, que con mayor o menor complejidad cuentan una historia. Por ejemplo, la campaña Beyond Scott, de MRM Meteorite London para Intel, permite al usuario seguir en tiempo real a un viajero que recrea el histórico viaje de Robert Falcon Scott a la Antártida. Pese a la dificultad de las comunicaciones, la aplicación se las ingenia para permitir ese seguimiento cruzando la ruta y los tiempos del explorador mediante geolocalización, el relato que este les transmite mediante un teléfono por satélite, y las imágenes obtenidas vía satélite, información metereológica, etc. El resultado es un relato inmersivo en tiempo real de la aventura de un expedicionario, favoreciendo esa idea de la marca como contadora - posibilitadora - de grandes historias que los usuarios pueden experimentar vicariamente y de las que pueden sentirse parte (Fernández Gómez, Rubio-Hernández \& Pineda, 2019). Otro caso sería el de la campaña \#GuiltyTags, de la agencia McCann Paris para la ONG Innocence in Danger. En ella, se narran 9 historias de asesinatos de niños - ficticios pero basados en hechos reales - a través de etiquetas en fotos de Instagram. Estas etiquetas conducen al perfil del asesino, pero también a los de todas aquellas personas próximas al niño pero que miraron hacia otro lado ante un posible caso de violencia. Si bien no existe aquí un caso tradicional de narrativa con presentación, nudo y desenlace, sí se puede encontrar una evolución narrativa que se traslada al usuario de forma hipertextual y que este deberá recomponer observando las distintas publicaciones del timeline de estos perfiles. 
Sin embargo, en la mayoría de campañas analizadas no existe esta transición entre estados y no puede observarse una historia en sentido estricto. Tendría lugar, en estos casos, una estructura más descriptiva que narrativa - aun cuando el Grupo de Entrevernes explica también que la narratividad está presente en mayor o menor medida en cualquier tipo de texto (1982) - . No es extraño encontrar esta visión más flexible acerca del storytelling en su aplicación al mundo de la publicidad, donde ya no es tan importante la construcción de un relato en el sentido tradicional como el paso de los fríos datos a una dimensión más humana y conectada con la experiencia del consumidor. Por ejemplo, la campaña Most dangerous street, de FCB Chicago para el Illinois Council Against Handgun Violence, intenta concienciar acerca del hecho de que se dispara a 40 personas al día en Chicago. Mientras que este dato frío y disperso a lo largo de la ciudad no resulta significativo para el ciudadano medio, condensar la representación de los 40 disparos en una misma calle resulta muy impactante. Así, la campaña muestra a través de láseres las trayectorias de las 40 balas, así como proyecciones en las paredes con datos sobre las víctimas. En otras ocasiones, la forma de dar volumen a estos datos es a través de infografías imaginativas de tipos diversos. Por ejemplo, Destination pride, de FCB/Six Toronto para Pflag Canada, visualiza datos sobre igualdad y derechos LGTBi empleando la bandera arcoiris como un gráfico de barras; y World of belonging, de Tool Santa Monica para Airbnb, muestra un atractivo mapa interactivo y animado en tiempo real con la actividad global de usuarios de Airbnb, con vistas a mostrar la envergadura de la plataforma y eliminar así suspicacias por parte de usuarios potenciales. Y si estas campañas humanizan datos, también hay campañas que directamente intentan humanizar un determinado servicio de datos, como sucede con Run that town, de Leo Burnett Sydney para promocionar el censo del Australian Bureau of Statistics. Esta campaña es un videojuego de gestión de una ciudad donde todos los datos provienen del censo, de modo que el público pueda comprender su utilidad de forma empírica. Como se ha dicho, si somos muy estrictos, aquí parece existir más un proceso de gamificación que de narración propiamente dicha. Incluso hay casos donde la campaña es casi una traducción ilustrativa o un proceso de inferencias a partir de los datos. Por ejemplo, The corruption converter, de FCB Brasil Sao Paulo para Estadao Newspaper, calcula qué beneficios en términos de servicios sociales - número de ambulancias, escuelas, vacunas... - implicarían los costes de la corrupción brasileña; o NRG solar forecast, de Droga5 New York para NRG, consiste en añadir a la predicción del tiempo -ya sea esta en televisión, en banners o en apps- lo que se podría hacer con la energía generada por el Sol ese día si se emplearan placas solares. La presencia de estas campañas es coherente con el esfuerzo que gobiernos y organizaciones vienen desarrollando por convertir en más accesibles y atractivos los datos públicos para la ciudadanía (Holland, 2019; Eberhardt y Silveira, 2018).

Un aspecto llamativo y que puede detectarse en la propia definición de la sección "Data Storytelling" por parte de Cannes Lions es que la relación entre datos y storytelling es inequívocamente bidireccional. Esto, lejos de ser una disquisición de carácter teórico, es algo con una clara plasmación empírica en las campañas analizadas. Así, existen, por una parte, campañas que emplean los datos como un recurso creativo para dinamizar el storytelling - que por sí mismo no es una novedad en el discurso publicitario-; y, por otra, campañas que emplean el storytelling como recurso creativo para dinamizar la exposición de datos. En cuanto al primer caso, campañas como Living memories, de Y\&R NZ Auckland para la 
organización benéfica de seguridad vial Brake NZ, emplean las posibilidades prestadas por los datos para contar una historia de un modo más sugerente. La mencionada campaña neozelandesa crea digitalmente imágenes de cómo serían niños fallecidos en accidentes de tráfico si siguieran vivos a día de hoy, con el consecuente impacto emocional en el espectador. Para mostrar dichas imágenes emplean técnicas forenses basadas en datos acerca de los rasgos probables del envejecimiento. El potencial de los datos y la tecnología es aquí un instrumento para realizar un aporte creativo a una historia sobre personas.

Figura 1. Fotograma de la campaña Living memories

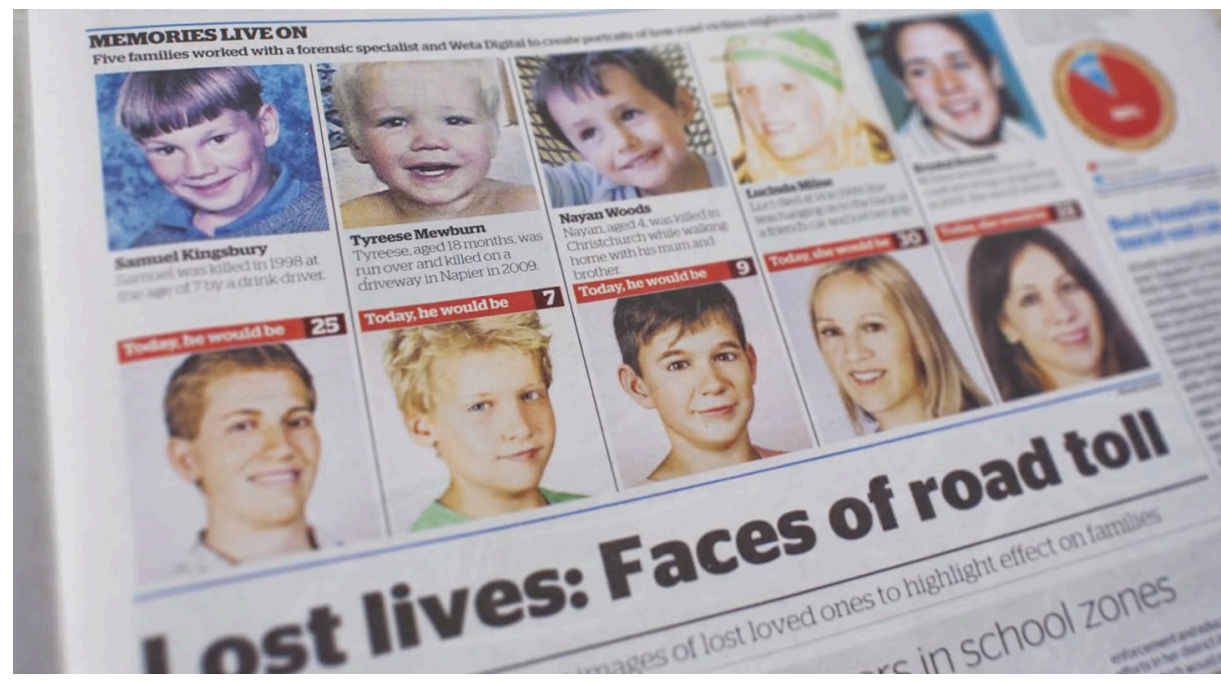

Dentro de este mismo grupo de campañas, pero con un uso muy diferente de los datos, la campaña Value of nature, de Deloitte Australia Melbourne para The Great Barrier Reef Foundation, parte de que la gente no entiende realmente el valor incalculable de la Gran Barrera de Coral australiana, así que la mejor forma de conseguir que lo hagan es calcular dicho valor incalculable, aportando datos cuantitativos sobre los beneficios económicos del mayor arrecife de coral del mundo.

En cuanto al segundo caso, el de campañas que emplean el storytelling como recurso creativo para humanizar la frialdad de los datos, podríamos citar campañas como Your year. Directed by Nike+, de AKQA Portland para Nike, o Spotify year in Music, de Razorfish New York para Spotify. Ambas emplean datos de los que disponen - por el uso de la aplicaciones Nike+ y Spofity - y los ofrecen al público de forma sugerente y atractiva empleando una dimensión narrativa. El caso de Nike, por ejemplo, intenta asemejarse a una pequeña película personalizada sobre las costumbres del usuario a la hora de hacer deporte; Spotify, por su parte, ofrece datos acerca de qué música ha escuchado el mundo -o un país concreto, o el usuario en cuestión- durante el año: qué canciones, qué géneros musicales, si más alegres o más tristes, etc. Otros ejemplos ya mencionados, como Most dangerous street sobre la representación de los disparos diarios de Chicago en una sola calle, o The corruption converter, sobre los beneficios que podrían haber generado las sumas perdidas por la 
corrupción brasileña, entrarían también aquí.

El tipo de datos es algo en lo que merece también la pena detenerse. En muchas ocasiones se hace equivaler datos a big data. Lo cierto es que numerosas campañas se apoyan, efectivamente, en big data o macrodatos. Por ejemplo, la campaña Google year in search, de R/GA London para Google, tiene como pieza central un vídeo - que deriva en un site que permite una mayor profundización- que recopila el año 2014 a partir de las búsquedas llevadas a cabo en Google. Existe aquí un procesamiento de datos complejo, basado en encontrar patrones repetitivos en conjuntos masivos de datos. Sin embargo, otras campañas no se basan en big data o macrodatos, sino en small data o microdatos, término que alude a una información más específica y manejable, y que permite en muchas ocasiones inferencias más útiles a la publicidad en términos de insights de consumidor (Lindstrom, 2016). La campaña \#IsltOkForGuys, de 72andSunny Amsterdam para Axe, analiza las búsquedas que realizan muchos chicos en Google para ver si algo «está bien para los chicos». A partir de esa evidencia de que muchos chicos están constreñidos por una masculinidad estereotipada y tóxica, la campaña reivindica el derecho a ser uno mismo a través de un spot, una plataforma online, etc. Con tanta frecuencia como los big o small data, que en ambos casos aluden a una forma de investigar al consumidor, muchas campañas de data storytelling parten simplemente de los datos individuales del propio usuario, como las mencionadas de Nike y Spotify que resumían el año del usuario. Pero, llamativamente, la gran mayoría de campañas analizadas no parten de datos del usuario - ni individuales ni compilados -, sino de datos históricos, estadísticos, etc. Por ejemplo, la campaña Meet Graham, de Clemenger BBDO Melbourne para Transport Accident Commission Victoria, se basa en la creación de una escultura que representa la forma que el ser humano debería tener para sobrevivir a todos los accidentes de tráfico. El resultado visual, tremendamente impactante, fue desarrollado por un cirujano de emergencias, un ingeniero de seguridad vial y una artista plástica usando datos estadísticos sobre seguridad vial, investigaciones médicas y creatividad. Por su parte, la campaña Every man remembered, de $R K C R / Y \& R$ London para la Royal British Legion, parte de datos históricos sobre los fallecidos en la Primera Guerra Mundial para desarrollar una gran base de datos con todos ellos en una suerte de homenaje en el que el público puede participar; y la campaña Reviving legends, de Dentsu Tokyo para el Japan Sport Council con motivo de la inminente demolición del National Stadium, convierte las hazañas de deportistas históricos en datos y las recrea visualmente a través de todo tipo de efectos visuales. Por supuesto, el tipo de datos no siempre tiene una única procedencia, y muchas campañas se basan en su cruce. Por ejemplo, el site de la campaña El tiempo que nos queda, de Leo Burnett Madrid para Ruavieja (Pernod Ricard), formula una serie de preguntas al usuario acerca de su relación con otra persona, y realizando algunos cálculos a partir de sus respuestas y cruzándolas con datos extraídos del Instituto Nacional de Estadística, le permite saber cuánto tiempo va a pasar con esa persona el resto de su vida. La campaña, lanzada a través de un spot muy emotivo, hace ver al usuario que, frente al uso intensivo que llevamos a cabo de la tecnología y las redes sociales, «tenemos que vernos más». Y lo hace con datos proporcionados por el propio usuario y procesados en relación con datos estadísticos.

El tópico cultural en torno al uso de los datos nos dibuja una automatización absoluta, donde la mano humana no es necesaria más que como garante de que todo sigue su camino. Sin 
embargo, lo cierto es que ni el procesamiento y la interpretación de datos, ni la elaboración de relatos y/o campañas publicitarias tienen sentido sin que el ser humano tome parte activa en el proceso. Así, cualquier campaña publicitaria parte del trabajo - estratégico, creativo, etc. de una agencia publicitaria, que se mantiene como pivote del trabajo publicitario también

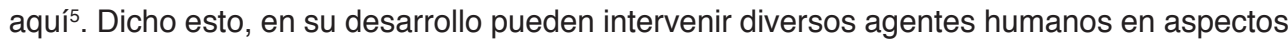
relacionados con la creatividad, la producción, la distribución o las relaciones públicas. Por ejemplo, de cara a la mencionada \#Guiltytags de la ONG Innocence in Danger, cooperan McCann Paris como agencia responsable, La Pac Paris, McCann Paris, MRM//McCann Paris, No Problemo Paris y The Paris como responsables de la producción; Media Transports IssyLes-Moulineaux como responsable de medios, y Weber Shandwick Paris como responsable de relaciones públicas. De hecho, el componente humano es absolutamente clave a la hora de trabajar con datos, como señala Yasuharu Sasaki, Head of Digital Creative y Director Creativo Ejecutivo en Dentsu y Presidente del Jurado de la categoría "Creative Data" en el festival Cannes Lions 2019:

\begin{abstract}
En el otro lado de nuestro mundo descansa otro mundo, llamado data. Cada movimiento individual de personas, cosas e información es recogido en este otro mundo [...] Pero este mundo de datos no es una bola de cristal [...] Quedarse mirándolo no te dirá nada [...] Alimentar la inteligencia artificial con más datos es inútil sin un plan [...] Sin embargo, si podemos conectar de algún modo estos datos previamente disgregados de maneras nuevas, inesperadas, creativas y solo humanas, estos pueden a veces revelar futuros increíbles (Cannes Lions, 201).
\end{abstract}

Dicho lo anterior, y aun asumiendo que la acción humana es esencial en la ideación y ejecución de una campaña de cualquier tipo, existen campañas en las que la dimensión narrativa es desarrollada directamente por la mano humana y otras en las que aquella es desarrollada de forma automatizada - aunque la programación de ese automatismo provenga necesariamente de un ser humano-. Un caso evidente en el que la acción humana es el elemento clave es la campaña Thanks 2016, it's been weird, de Spotify, que parte de datos extraños obtenidos del uso de los usuarios y los transforma en gráficas y vallas con titulares como "Querida persona que reprodujo "Sorry" 42 veces el día de San Valentín, ¿qué hiciste?» O «A los 1.235 chicos que siguieron la playlist "Noche de chicas" este año, os queremos". La mano humana es evidente en la campaña Snelweg sprookjes ["Cuentos de la Carretera"], de ISOBAR Amsterdam para Volkswagen. Esta parte de que los niños pasan sus trayectos en coche mirando dispositivos móviles y no disfrutando del paisaje o ejercitando su imaginación. La campaña consiste, pues, en una aplicación móvil que les habla y construye micronarrativas en relación con el lugar exacto por el que el coche está pasando, dando lugar a una especie de colección de cuentos geolocalizados. La geolocalización implica un proceso automatizado, pero las micronarrativas específicamente asociadas a los distintos lugares fueron creadas al comienzo por una serie de escritores infantiles. Puede apreciarse una mayor automatización en la campaña AiMEN, de BETC Paris para promocionar la serie

5/ No obstante, hay que mencionar que entre las agencias creadoras de estas campañas se encuentran algunas como Anomaly o R/GA, que desbordan el concepto tradicional de agencia publicitaria (SelvaRuiz, 2019: 190). 
The young pope de Canal +. La campaña se basa en que el Papa de la ficción responde en tiempo real con versículos de la Biblia a tweets de personas totalmente ajenas a la ficción. La elección de los tweets que serían respondidos y la del versículo concreto para el tweet de respuesta correspondía a la inteligencia artificial ${ }^{6}$, y eso permitió que en dos semanas se analizaran casi 4 millones y medio de mensajes, lo cual implica $10 \mathrm{~GB}$ de texto, y se respondiera a 900.000 usuarios tomando alguno de los 39.000 versículos de la Biblia. Esta campaña, que tomó una media de 2 segundos para el análisis de cada tuit, habría sido sencillamente imposible sin el empleo de la inteligencia artificial (Desbois, 2017).

Figura 2. Fotograma de la campaña AiMEN

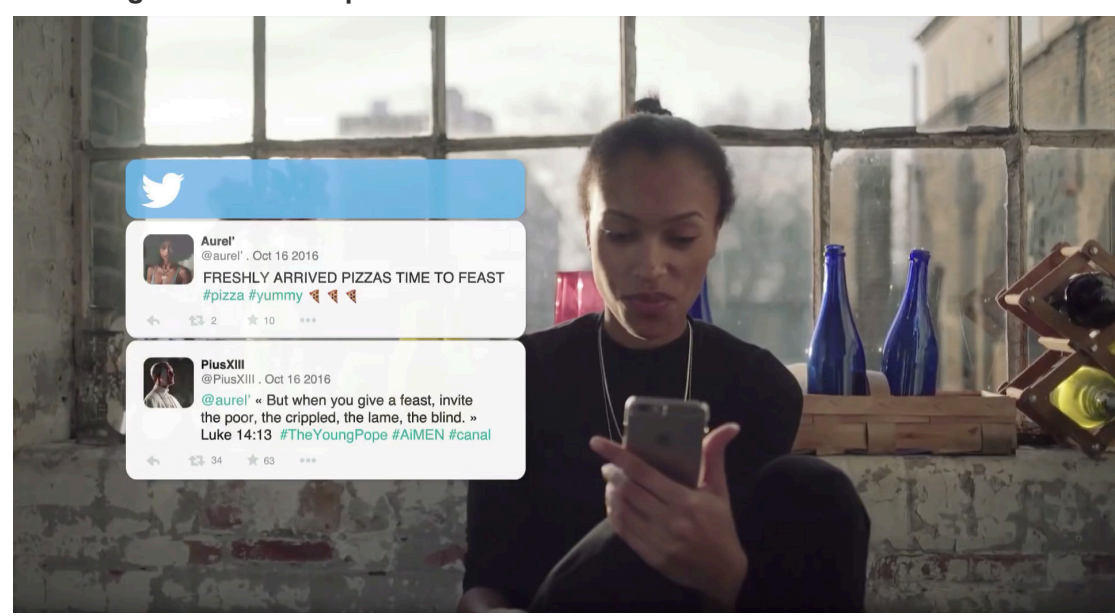

Existen numerosos recursos tecnológicos empleados por las campañas analizadas en relación con la recopilación de datos, la plasmación creativa/narrativa de la campaña, o la difusión de esta. El rasgo clave que puede destacarse en este sentido es la diversidad y, a la vez, la interconexión entre estos recursos y procedimientos. Es habitual encontrar que en estas campañas confluyan, por ejemplo, geolocalización, inteligencia artificial, big data, realidad aumentada, y procesos de inteligencia colectiva. En cuanto a herramientas, encontramos desde piezas gráficas o audiovisuales hasta advergames y simuladores diversos, alternate

\footnotetext{
6/ La productora Make Me Pulse Paris empleó el IBM Watson Natural Language Classifier, algoritmos de aprendizaje automático y un analizador de sentimientos para interpretar el tono de los tweets de los usuarios y darles una respuesta relevante (Desbois, 2017).
} 
reality games $(\mathrm{ARG})^{7}$, o extensiones para el navegador ${ }^{8}$, y pasando por infografías, banners, apps, webs, etc. No solo se trata de que estas tecnologías y herramientas vinculadas a medios diversos puedan coexistir en una misma campaña, sino que en muchas ocasiones la interacción entre ellos es el motor creativo de la campaña. Incluso resulta fácil observar cómo se interrelacionan en estas campañas lo above y lo below the line, lo físico y lo digital, etc. Por ejemplo, resulta imposible ubicar en clasificaciones tradicionales casos como Bittersweet pies, campaña de bajo presupuesto de McCann Worldgroup Romania Bucharest para los restaurantes Paul, que consiste principalmente en la creación de tartas (reales) como gráficos sobre la baja representación de la mujer en ciertas parcelas de la sociedad, para luego desarrollar también piezas digitales y de exterior. También inclasificable resulta Google home of the Whopper, de David Miami para Burger King, consistente en el lanzamiento de un spot de televisión pensado para trolear al asistente de voz de Google, al preguntarle qué es un Whopper, con el resultado de que los asistentes de voz de los hogares de los espectadores leen en voz alta por sorpresa la definición de Whopper extraída de Wikipedia. O, por último, The emotional trailer, de McCann Melbourne para el Melbourne International Film Festival, que emplea máquinas de electroestimulación para generar emociones faciales en determinados sujetos de forma artificial. El "tráiler emocional" implica ver a la persona cubierta de electrodos gesticulando con las emociones que la película supuestamente despertaría en ella.

Pero incluso, yendo más allá, cabe observar que muchas de estas campañas son más bien proyectos que se centran en aportar valor al usuario. No solo no son, sin más, mensajes publicitarios emitidos a través de medios de comunicación, sino que pretenden ser contenidos útiles o entretenidos para el usuario hasta el punto de que en muchos casos incluso estarían más próximos a la definición de producto que a la de comunicación. Dos casos muy evidentes en este sentido serían Project revoice y Yournalist. El primero, de BWM Dentsu Sydney para The ALS Association, se centra en personas con esclerosis lateral amiotrófica (ELA, o ALS en inglés) que han perdido su voz real y tienen que hablar a través de un ordenador -el caso de Stephen Hawking puede ser el más conocido de esta enfermedad-. Project revoice emplea un software que analiza unas pocas horas de la voz real del enfermo y recrea su voz para que la emitida por el ordenador sea exactamente la que tenía antes de perderla. En el segundo caso mencionado, Yournalist, proyecto de la agencia The Cyranos//McCann Barcelona para EITB (la radiotelevisión pública vasca), se centra en las noticias falsas y en la polarización habitual que surge de que los ciudadanos se informen únicamente por la fuente

7/ Los alternate reality games o juegos de realidad alternativa (ARG) son narraciones participativas que, aun siendo habitualmente ficticias, emplean el mundo real como soporte y requieren de la participación activa del público para avanzar. Explicándolo de forma sencilla, vendría a ser algo así como una gymkhana o un juego de rol de carácter transmedia. Las campañas Project Architeuthis, de Lowe Campbell Ewald Detroit para la US Navy, y Geeks go for \#CC9900, de Clemenger BBDO Sydney para General Electric, van en esta línea, con puzles tremendamente complejos para los que serían necesarios conocimientos muy especializados.

8/ Por ejemplo, la campaña S.H.E., de Grey West Los Angeles para Pantene (P\&G), proporciona una extensión para el navegador, de forma que los resultados de búsqueda sean más igualitarios en temas de género, evitando sesgos sexistas y dando visibilidad a las mujeres. 
más próxima a su cosmovisión y no puedan, por tanto, contrastar fuentes. La propuesta es una aplicación que, para un mismo hecho, permite conocer las noticias provenientes de 6 fuentes diversas, de forma que la persona pueda ver todas las caras de aquel. En ambos casos, parece evidente que se va mucho más allá de lo que tradicionalmente se consideraba una campaña publicitaria tradicional, e incluso más allá de los medios no convencionales. Se ofrecen directamente productos y servicios que proporcionan valor al usuario, y que pueden observarse como una versión más avanzada de la estrategia de branded content que lograría realmente superar la noción de publicidad y comunicación para ofrecer un producto o servicio al usuario (Wagler, 2013).

Si esta última consideración respecto a las campañas analizadas permite observar una tendencia que, aunque cada vez más consolidada, puede considerarse novedosa respecto a la publicidad tradicional, a continuación puede observarse una que la conecta con usos asentados en el sector publicitario. Se trata de que una gran parte de las campañas analizadas muestran una relación, por una parte, con insights y experiencias cotidianas, y por otra, con la agenda pública. Esto no debería ser una novedad, como no debe sorprender que en el proceso de trabajo se parta de un brief, se alcance una big idea, etc. No obstante, resulta importante destacar que los datos no solo no parecen estar enfriando la creatividad, sino que están potenciando nuevas formas de conectar y comunicarse de forma relevante con el consumidor. En cuanto a los insights, hay que partir de que los datos pueden ser, de hecho, una forma de obtenerlos. Pero, además, pueden ayudar a que una idea sea comunicada de forma sugerente al público. Por ejemplo, la campaña Spotify music for your world de Anomaly Toronto para Spotify Canada, se basa en la creación de playlists con fuertes referencias a barrios o ciudades concretas y marcadas por insights que intentan conectar con el potencial usuario canadiense, en un intento de penetrar en un mercado que le era esquivo. Estas playlists eran además difundidas con una estrategia totalmente localizada. Por su parte, la campaña Tagwords, de Africa São Paulo para Budweiser, se basa en un insight muy potente de partida: Budweiser ha estado presente en la historia musical de EEUU, y hay infinidad de fotos de músicos relevantes consumiéndola. El problema es que estas fotos no podrían ser usadas en la publicidad de la marca sin tener que desembolsar enormes cifras de dinero. La solución es el lanzamiento de piezas de publicidad exterior, gráfica y banners en las que se indican las palabras que el usuario debe usar en Google para encontrar la fotografía en cuestión. Y el resultado es muy potente a la hora de asociar Budweiser con la música y muy sugerente en su forma de llevarlo a cabo. Asimismo, muchas de las campañas analizadas muestran una relación con la agenda pública. Un ejemplo sería Hotel Cup, campaña de Johannes Leonardo New York para Tripadvisor durante la Copa Mundial de Brasil 2014, la cual, emulando la tendencia habitual a que animales realicen predicciones acerca de los ganadores en los partidos de los mundiales de fútbol, propone un método supuestamente más fiable: predice los resultados de cada partido en función de la puntuación media de los usuarios de Tripadvisor del hotel en que se aloja cada selección.

Un rasgo bastante llamativo presente en un alto número de las campañas analizadas es un enfoque metadiscursivo sobre los datos y, en general, la tecnología. Es decir, serían campañas basadas en datos que reflexionan sobre los datos. No en vano, muchos case studies presentados por estas agencias a festivales argumentan como un punto fuerte el haber generado conversación o debate acerca de los datos y la tecnología. Este enfoque 
metadiscursivo puede tener tanto un tono celebratorio como uno crítico, pero tiene lugar en numerosos casos. Para colmo, hay varias campañas desarrolladas por empresas que se dedican abiertamente a los datos - en particular, Google cuenta con varias campañas en la muestra analizada - o, al menos, son compañías digitales que recopilan numerosos datos por la propia actividad de los usuarios - Spotify, Tripadvisor, etc. - . Hay dos casos particularmente interesantes en esta metadiscursividad. El primero es The next Rembrandt, de J. Walter Thompson Amsterdam para ING, que consiste en la creación de una nueva obra pictórica de Rembrandt. Para ello, se analizan 346 cuadros de Rembrandt de forma automatizada durante 18 meses, tanto en cuanto a los aspectos figurativos - rostros, posiciones, luces, colores, etc. - como en cuanto a los técnicos - tipo de trazos, volumen de la pintura, etc. - y el propio software, partiendo de dichos datos y con la ayuda de una impresora 3D, crea un cuadro totalmente nuevo de Rembrandt y sorprendentemente verosímil. Esto generó un debate - potenciado por la propia campaña - acerca de las posibilidades de la tecnología y la disolución de fronteras entre esta y la humanidad. La segunda campaña que ejemplifica especialmente esta metadiscursividad es Predictive world, de Sid Lee Paris para la promoción del videojuego Watch Dogs 2 de Ubisoft. Desde una perspectiva abiertamente crítica, la campaña proporciona un complejo perfil del propio usuario similar al que podría disponer de él una empresa, y lo hace tomando datos provenientes de diversas fuentes disponibles de manera automatizada.

\section{Conclusiones}

Se han podido observar a lo largo de este trabajo las enormes potencialidades del data storytelling de cara a la creación de campañas atractivas y significativas para el usuario, así como el uso actual que numerosas organizaciones están haciendo ya de él. Si solo 5 años después de la creación de la categoría "Creative Data" en el festival Cannes Lions pueden ya encontrarse usos creativos de los datos de tanto calibre y diversidad, resulta difícil imaginar hasta dónde pueden llegar los avances en este sentido.

Dicho esto, igual que es fácil entrever las potencialidades del data storytelling, resulta sencillo también entender que estamos en un estadio muy primario de su desarrollo, así como de su investigación académica. Muchos de los trabajos que han servido como base teórica a este artículo abordan temas mucho más parciales y, a la vez, menos actuales que este, lo cual provoca que las clasificaciones preexistentes no resulten del todo útiles para encuadrar las campañas más exitosas de data storytelling de los últimos 5 años. Si autores como Hook (2018) investigaban el uso de datos en vídeo, las campañas analizadas nos muestran una diversidad extrema de tecnologías, herramientas y medios que hace muy difícil la aplicación de su clasificación. Más cerca pueden estar las conclusiones de este trabajo de las de Holland (2019) acerca de las formas de contar historias con datos.

En cualquier caso, una de las conclusiones principales que pueden extraerse de este trabajo es que el propio concepto de data storytelling es amplio, complejo y diverso. Así, caben en él tanto historias con presentación, nudo y desenlace como infografías atractivas; tanto big data como small data; tanto datos individuales de un usuario como datos históricos 
obtenidos de una base de datos pública; tanto campañas desarrolladas íntegramente mediante acción humana como otras cuyo desarrollo recae, por ejemplo, en inteligencia artificial; tanto tecnologías muy avanzadas como herramientas publicitarias convencionales; y, sobre todo, tanto campañas en las que los datos son empleados como recurso creativo para enriquecer el storytelling de la marca como campañas en las que el storytelling es utilizado como recurso creativo para humanizar y hacer relevantes los datos.

Se comprueba, pues, que la situación actual es la de un contexto de cambio en el que la clave es la novedad y la experimentación sin reglas o dogmas prefijados. Un indicio de esta juventud del data storytelling puede encontrarse en la marcada tendencia a la metadiscursividad observada en las campañas analizadas, que hablan sobre datos, proponen debates acerca de la tecnología, son firmadas en muchas ocasiones por empresas que gestionan grandes volúmenes de datos, etc. $Y$ aun así, no todo ha cambiado: siguen existiendo rutinas de trabajo próximas a las de la publicidad tradicional, y al mismo tiempo la agencia parece estar sabiendo evolucionar para adaptarse a este nuevo ecosistema (Stuhlfaut y Windels, 2019). $Y$ al tiempo se consolidan tendencias que cada vez dejan de ser menos novedosas, como la aportación de valor al usuario hasta el punto de que muchas campañas están a medio camino entre la comunicación y el producto, en lo que puede observarse como una tendencia que superaría la estrategia del branded content para abrir nuevas líneas de producto/servicio.

Dada la cantidad, la envergadura y la relevancia de las campañas seleccionadas por Cannes Lions, no cabe duda de que los datos y los relatos asociados a ellos constituyen un fenómeno que ha venido para quedarse y que no solo puede no constreñir la creatividad en el discurso publicitario, sino que incluso puede potenciarla añadiendo nuevos elementos a la ecuación.

\section{Bibliografía}

ÁlVAREZ MONZONCILLO, José María (2013). Las nuevas televisiones. Personalización e individualización. Comunicación: estudios venezolanos de comunicación, (163), 114-125. Recuperado de https://dialnet.unirioja.es/servlet/articulo?codigo=6027116

BOYD, DANA y CRAWFORD, Kate (2012). Critical questions for big data. Information, communication \& society, 15(5), 662-679. doi: 10.1080/1369118X.2012.678878

CANNES LIONS (2019a). Creative data lions. Canneslions.com. Recuperado de https://www.canneslions. com/enter/awards/reach/creative-data-lions

CANNES LIONS (2019b). 5 lecciones (atronadoras) escuchadas en la última edición de Cannes Lions. Marketingdirecto.com, 25-06-2019. Recuperado de https://www.marketingdirecto.com/especiales/ cannes-lions/5-lecciones-atronadoras-escuchadas-ultima-edicion-cannes-lions

CARO-CASTAÑO, Lucía y SELVA-RUIZ, David (2011). Estrategias de convergencia y desintermediación en la difusión del videoclip mediante autocomunicación de masas. Trípodos, n. extra, 155-163. 
CARRILLO-DURÁN, María-Victoria y RODRÍGUEZ-SILGADO, Ana (2018). El ecosistema programático. La nueva publicidad digital que conecta datos con personas. El profesional de la información, 27(1), 195-201. doi: 10.3145/epi.2018.ene.18

COULDRY, Nick y TUROW, Joseph (2014). Advertising, big data and the clearance of the public realm: marketers' new approaches to the content subsidy. International journal of communication, 8 , 1710-1726. Recuperado de https://ijoc.org/index.php/ijoc/article/view/2166

CUFOGLU, Ayse (2014). User profiling-a short review. International journal of computer applications, 108(3), 1-9. Recuperado de https://pdfs.semanticscholar.org/eecb/ f9358916a8e7db20511c611eaceaac554417.pdf

DAHLEN, Micael y ROSENGREN, Sara (2016). If advertising won't die, what will it be? Toward a working definition of advertising. Journalofadvertising, 45(3), 334-345. doi:10.1080/00913367.2016.1172387

DESBOIS, Juliette (2017). The young pope - AiMEN: Trolling the internet as a pontifical artificial intelligence. Makemepulse.com. Recuperado de https://m.makemepulse.com/the-young-popeaimen-37ab94d8bf83

EBERHARDT, André y SILVEIRA, Milene Selbach (2018). Show me the data!: A systematic mapping on open government data visualization. En A. Zuiderwijk y C. C. Hinnant (Eds.), Proceedings of the 19th Annual International Conference on Digital Government Research: Governance in the Data Age (artículo 33). New York: ACM. doi: 10.1145/3209281.3209337

FENG, Songming y OTS, Mart (2018). Seeing native advertising production via the business model lens: The case of Forbes's BrandVoice Unit. Journal of interactive advertising, 18(2), 148-161. doi: 10.1080/15252019.2018.1491349

FERNÁNDEZ GÓMEZ, Jorge David, RUBIO-HERNÁNDEZ, María-del-Mar y PINEDA, Antonio (2019). Branding cultural. Una teoría aplicada a las marcas y a la publicidad. Barcelona: UOC.

GILMORE, James N. (2016). Everywear: The quantified self and wearable fitness technologies. New media \& society, 18(11), 2524-2539. doi: 10.1177/1461444815588768

GÓMEZ NIETO, Begoña (2018). El influencer: herramienta clave en el contexto digital de la publicidad engañosa. Methaodos, 6(1), 149-156. doi: 10.17502/m.rcs.v6i1.212

GONZÁLEZ, Roberto J. (2017). Hacking the citizenry?: Personality profiling,'big data'and the election of Donald Trump. Anthropology today, 33(3), 9-12. doi: 10.1111/1467-8322.12348

GRASSEGGER, Hannes y KROGERUS, Mikael (2017). The data that turned the world upside down. Vice.com, 28-01-2017. Recuperado de: https://www.vice.com/en_us/article/mg9vvn/how-our-likeshelped-trump-win 
GRUPO DE ENTREVERNES (1982). Análisis semiótico de los textos: introducción, teoría, práctica. Madrid: Ediciones Cristiandad.

HOLLAND, Taylor (2019). From stats to stories: The evolution of data storytelling. Skyword.com, 20-032019. Recuperado de https://www.skyword.com/contentstandard/creativity/from-stats-to-storiesthe-evolution-of-data-storytelling

HOOK, Jonathan (2018). Facts, interactivity and videotape: exploring the design space of data in interactive video storytelling. En Proceedings of the 2018 ACM International Conference on Interactive Experiences for TV and Online Video(43-55). New York, USA: ACM. doi: 10.1145/3210825.3210826

JUNG, A-Reum (2017). The influence of perceived ad relevance on social media advertising: An empirical examination of a mediating role of privacy concern. Computers in human behavior, 70, 303-309. doi: 10.1016/j.chb.2017.01.008

LINDSTROM, Martin (2016). Small data. Las pequeñas pistas que nos advierten de las grandes tendencias. Madrid: Deusto.

MARWICK, Alice E. (2013). Status update: Celebrity, publicity, and branding in the social media age. New Haven/London: Yale University Press.

MAVRIKI, Paola y KARYDA, Maria (2017). Using personalization technologies for political purposes: Privacy implications. En Sokratis K. Katsikas, and Vasilios Zorkadis (Eds.). International Conference on e-Democracy -Privacy-Preserving, Secure, Intelligent E-Government Services (33-46). Cham: Springer.

MAYER-SCHÖNBERGER, Viktor y CUKIER, Kenneth (2013). Big data. A revolution that will transform how we live, work, and think. London: John Murray Publishers.

MCKEE, Robert y GERACE, Thomas (2018). Storynomics: Story-driven marketing in the post-advertising world. New York: Twelve..

MCLUHAN, Marshall (1980). Comprender los medios de comunicación: las extensiones del ser humano. Barcelona: Paidós. Recuperado de https://cedoc.infd.edu.ar/upload/McLuhan_Marshall_ Comprender_los_medios_de_comunicacion.pdf

MCSTAY, Andrew (2016). Empathic media and advertising: Industry, policy, legal and citizen perspectives (the case for intimacy). Big data \& society, 3(2), 1-11. doi: 10.1177/2053951716666868

MILLER, Daniel y SINANAN, Jolynna (2014). Webcam. Malden: John Wiley \& Sons.

ROBERTS, Kevin. (2005). Lovemarks, el futuro más allá de las marcas. Madrid: Empresa Activa. 
SALMON, Christian (2008). Storytelling. La máquina de fabricar historias y formatear las mentes. Barcelona: Península.

SELVA-RUIZ, David (2019). Especies publicitarias. Perfiles profesionales en las agencias. Barcelona: Editorial UOC.

SELVA-RUIZ, David y CARO-CASTAÑO, Lucía (2016). Uso de datos en la creatividad publicitaria: el caso de Art, Copy \& Code de Google. El profesional de la información, 25(4), 642-651. doi: 10.3145/ epi.2016.jul.14

SOLANA, Daniel (2012). Postpublicidad. Reflexiones sobre una nueva cultura publicitaria en la era digital. Barcelona: Doubleyou.

STUHLFAUT, Mark W. u WINDELS, Kasey (2019). Altered states: The effects of media and technology on the creative process in advertising agencies. Journal of marketing communications, 25(1), 1-27. doi: 10.1080/13527266.2017.1380069

TRUONG, Yann, MCCOLL, Rod y KITCHEN, Philip (2010). Practitioners' perceptions of advertising strategies for digital media. International journal of advertising, 29(5), 709-725. doi: 10.2501/ S0265048710201439

TUROW, Joseph. (2011). The daily you: How the new advertising industry is defining your identity and your worth. New Haven/London: Yale University Press.

VAN DIJCK, Jose (2014). Datafication, dataism and dataveillance: Big Data between scientific paradigm and ideology. Surveillance \& society, 12(2), 197-208. doi: 10.24908/ss.v12i2.4776

VAN DIJCK, Jose y POELL, Thomas (2016). Understanding the promises and premises of online health platforms. Big data \& society, 3(1), 1-11. doi: 10.1177/2053951716654173

VAN DIJCK, Jose y POELL, Thomas (2013). Understanding social media logic. Media and communication, 1(1), 2-14. Recuperado de http://ssrn.com/abstract=2309065

WAGLER, Adam (2013). Embracing change: Exploring how creative professionals use interactive media in advertising campaigns. Journal of interactive advertising, 13(2), 118-127. doi: $10.1080 / 15252019.2013 .833001$

ZHU, Yu-Qian y CHANG, Jung-Hua (2016). The key role of relevance in personalized advertisement: Examining its impact on perceptions of privacy invasion, self-awareness, and continuous use intentions. Computers in human behavior, 65, 442-447. doi: 10.1016/j.chb.2016.08.048 
